\title{
INDIRECT RKKY-TYPE INTERACTION BY DIRECT OXYGEN HOPPING
}

\author{
J.J. RODRIGUEZ-NUÑEZ* $†$ AND B. COQBLIN \\ Université Paris-Sud, Laboratoire de Physique des Solides \\ Bâtiment 510, 91405 Orsay, Cedex, France

\section{H. BECK} \\ Université de Neuchâtel, Institut de Physique \\ Rue A.L. Breguet 1, 2000 Nenchâtel, Switzerland

\section{AND J. KONIOR} \\ Institute of Physics, Jagiellonian University, Reymonta 4, 30-059 Kraków, Poland
}

The three-band Emery model, describing the holes in the $\mathrm{CuO}_{2}$ planes of the high-temperature superconducting oxides, is considered. The model includes the direct oxygen-oxygen hopping integral $t_{p p}$. The exact Bogolyubov transformation is used to exclude one oxygen band and obtain a two-dimensional Anderson model. Afterward, the effective Hamiltonian is obtained by eliminating the second oxygen band with the use of two approximate canonical transformations. The effective Hamiltonian describes the spins residing on the copper sites and interacting through an indirect interaction $J_{\mathrm{SX}}(R)$, where $R$ is the distance between two copper ions. $J_{\mathrm{SX}}(R)$ depends on the doping rate $\delta$ and is a decaying function of $R$. Numerical results for $J_{\mathrm{SX}}(R)$ are given for different doping rates $\delta$ for the case of parabolic bands. The obtained interaction $J_{\mathrm{Sx}}(R)$, when added to the original antiferromagnetic interaction (present in oxides at $\delta=0$ ), might lead to a frustration of the long-range antiferromagnetic ordering upon doping.

PACS numbers: $74.20 . \mathrm{Mn}, \mathbf{7 4 . 2 5 . J b}$

*Permanent address: Departamento de Física, Grupo de Sólidos, Facultad Experimental de Ciencias, Universidad del Zulia, Apartado 526, Maracaibo, Estado Zulia, Venezuela.

$\dagger$ Also a Visiting Scientist at IVIC, Centro de Física, Apartado 21827, Caracas 1020-A, Venezuela. 


\section{Introduction}

It is now generally accepted that due to a short coherence length along the $c$-axis, the basic physics of the high-temperature copper oxides may be considered as restricted to the $\mathrm{CuO}_{2}$ planes, at least in the first approximation. The very beginning approaches for studying the electronic structure of the $\mathrm{CuO}_{2}$ planes were restricted to effective one-band Hamiltonians, i.e., the Hubbard [1] and the $t-J$ [2] models. After more decisive experimental results had become available, it became clear that one-band models were not sufficient to capture the richness of physical phenomena that were taking place in the cuprates. Emery [3] proposed to use the so-called three-band Hubbard model for describing the electronic structure of the $\mathrm{CuO}_{2}$ planes.

In this paper we study the Emery model of the $\mathrm{CuO}_{2}$ planes with the direct oxygen-oxygen hopping $t_{p p} \neq 0$. Since there are two oxygen atoms in an elementary cell, there are two oxygen bands. By performing the Bogolyubov transformation, we decouple one oxygen band and end up with the one-conduction-band Anderson model. To derive the effective Hamiltonian $H_{\text {eff }}$, we apply two canonical transformations. First, the Schrieffer and Wolff (SW) [4] transformation is used to eliminate the hybridization between copper and oxygen orbitals. A second transformation is used to obtain the long range interaction between coppers. As a result, $H_{\text {eff }}$ [Eq. (13)] may be written as a spin Hamiltonian containing only the localized copper spins with the indirect RKKY-type interaction $J_{\mathrm{SX}}(R)$, where $R$ is the separation between two copper ions. We show that $J_{\mathrm{SX}}(R)$ strongly depends on a doping rate of the $\mathrm{CuO}_{2}$ plane, $\delta$. We argue that the derived interaction $J_{\mathrm{SX}}(R)$ should be added to the original antiferromagnetic (AF) interaction already present in the parent compounds, i.e., in those with $\delta=0$. Since $J_{\mathrm{SX}}(R)$ becomes stronger with increasing doping rate $\delta$, then the disappearance of the long-range AF ordering upon doping might be due to the increased strength of $J_{\mathrm{Sx}}(R)$.

The paper is organized as follows. In Sec. 2 the three-band (Emery) model is introduced, one oxygen band is decoupled from the problem, and the effective Hamiltonian is derived with the use of two canonical transformations. Section 3 presents numerical values of $J_{\mathrm{SX}}(R)$ calculated for different doping rates $\delta$ for the case of parabolic bands. Section 4 discusses the obtained results and presents the final conclusions.

\section{The physical system}

The two-dimensional three-band Emery model [3] is believed to contain the essential ingredients of the electronic structure of superconducting oxides, at least in the first approximation. The model includes the $d_{x^{2}-y^{2}}$ orbitals of the copper ions located at sites $\boldsymbol{R}_{\boldsymbol{i}}$ of a two-dimensional quadratic lattice (with the lattice constant equal to $a)$, and the $p_{\sigma}$ orbitals centered at $\boldsymbol{R}_{i}+a \hat{x} / 2\left(p_{\sigma}=p_{x}\right)$ and at $\boldsymbol{R}_{i}+a \hat{\boldsymbol{y}} / 2\left(p_{\sigma}=p_{y}\right)$. We assume the lattice constant $a$ to be the unit of length, i.e., $a \equiv 1$ from now on. With a suitable phase convention [6], the Hamiltonian of the Emery model may be written as

$$
H=\left(\varepsilon_{d}-\mu\right) \sum_{i, \sigma} n_{i, \sigma}^{d}+\left(\varepsilon_{p}-\mu\right) \sum_{i, \sigma}\left(n_{i+\hat{x} / 2, \sigma}^{p}+n_{i+\hat{y} / 2, \sigma}^{p}\right)
$$




$$
\begin{aligned}
& +U_{d} \sum_{i} n_{i, \uparrow}^{d} n_{i, \downarrow}^{d}+U_{p} \sum_{i}\left(n_{i+\hat{x} / 2, \uparrow}^{p} n_{i+\hat{x} / 2, \downarrow}^{p}+n_{i+\hat{y} / 2, \uparrow}^{p} n_{i+\hat{y} / 2, \downarrow}^{p}\right) \\
& +t_{p d} \sum_{i, \sigma}^{i} \sum_{\alpha= \pm 1}\left[d_{i, \sigma}^{\dagger}\left(-\alpha p_{i+\alpha \hat{x} / 2, \sigma}+\alpha p_{i+\alpha \hat{y} / 2, \sigma}\right)+\text { H.c. }\right] \\
& +t_{p p} \sum_{i, \sigma}^{i, \sigma} \sum_{\alpha, \beta= \pm 1}^{\alpha= \pm 1}\left[(\alpha \beta) p_{i+\alpha \hat{x} / 2, \sigma}^{\dagger} p_{i+\beta \hat{y} / 2, \sigma}+\text { H.c. }\right] \text {. }
\end{aligned}
$$

where $\varepsilon_{d}\left(\varepsilon_{p}\right)$ is the local-orbital energy level for the copper- $d_{x^{2}-y^{2}}$ (oxygen- $p_{\sigma}$ ) orbital, $U_{d}\left(U_{p}\right)$ is the copper (oxygen) Hubbard coupling, and $\mu$ is the chemical potential. The corresponding fermion annihilation and creation operators are denoted $d_{i, \sigma}$ and $d_{i, \sigma}^{\dagger}$ for the $d_{x^{2}-y^{2}}$ orbital centered at $\boldsymbol{R}_{i}$, while $p_{i+\hat{\alpha} / 2, \sigma}$ and $p_{i+\hat{\alpha} / 2, \sigma}^{\dagger}$ symbols are used for the corresponding $p_{\sigma}$ orbitals $(\alpha=x$ or $y$ ). Finally, the last two terms in the Hamiltonian describe the copper-oxygen and oxygen-oxygen hopping processes with the hopping amplitudes $t_{p d}$ and $t_{p p}$, respectively. Now, the oxygen fermion operators are transformed to the Fourier space

$$
p_{i+\hat{\alpha} / 2, \sigma}=\frac{1}{\sqrt{N}} \sum_{k} \exp \left[-\mathrm{i} \boldsymbol{k} \cdot\left(\boldsymbol{R}_{i}+\hat{\alpha} / 2\right)\right] p_{\alpha, k ; \sigma} .
$$

The result is

$$
\begin{aligned}
H= & \sum_{\nu=1,2} \sum_{k, \sigma} \varepsilon_{\nu, k ; \sigma} p_{\nu, k ; \sigma}^{\dagger} p_{\nu, k ; \sigma}+\left(\varepsilon_{d}-\mu\right) \sum_{i, \sigma} n_{i, \sigma}^{d}+U_{d} \sum_{i} n_{i, \uparrow}^{d} n_{i, \downarrow}^{d} \\
& +\frac{t_{p d}}{\sqrt{N}} \sum_{\nu=1,2} \sum_{i, k, \sigma}\left(\gamma_{\nu, k} \mathrm{e}^{-\mathrm{i} \boldsymbol{k} \cdot \boldsymbol{R}_{i}} p_{\nu, k ; \sigma}^{\dagger} d_{i, \sigma}+\text { H.c. }\right)
\end{aligned}
$$

where the two oxygen bands (with the indices $\nu=1$ and $\nu=2$ ) have been obtained instead of the original oxygen orbitals $p_{x}$ and $p_{y}$. The oxygen band quasiparticle energies and the hybridization coefficients are given by

and

$$
\varepsilon_{\nu, k ; \sigma}=\left(\varepsilon_{p}-\mu\right)-4 t_{p p}(-1)^{\nu} \sin \left(k_{x} / 2\right) \sin \left(k_{y} / 2\right),
$$

$$
\gamma_{\nu, k}=\sqrt{2}\left[\sin \left(k_{x} / 2+(-1)^{\nu} \sin \left(k_{y} / 2\right)\right],\right.
$$

respectively, and $N$ is the number of lattice sites in the system. We have neglected the Coulomb repulsion on the oxygen orbitals, i.e., $U_{p}=0$. This is justified for a low number of doped holes. To simplify the calculations, we perform a Bogolyubov rotation given by

where

$$
c_{k, \sigma}=\gamma_{k}^{-1} \widetilde{M}_{k} \phi_{k, \sigma}
$$

$$
\begin{aligned}
& c_{k, \sigma}=\left[c_{1, k ; \sigma}, c_{2, k_{; \sigma}}\right]^{T}, \\
& \phi_{k, \sigma}=\left[\phi_{1, k_{;} \sigma}, \phi_{2, k_{;} \sigma}\right]^{T}, \\
& \gamma_{k}^{2}=\gamma_{1, k}^{2}+\gamma_{2, k}^{2}=4\left[\sin ^{2}\left(k_{x} / 2\right)+\sin ^{2}\left(k_{y} / 2\right)\right],
\end{aligned}
$$

and the matrix $\widetilde{M}$ is given by

$$
\widetilde{M}_{k}=\left[\begin{array}{rr}
\gamma_{1, k} & \gamma_{2, k} \\
\gamma_{2, k} & -\gamma_{1, k}
\end{array}\right] \text {. }
$$


The Hamiltonian (1) is transformed to

with

$$
\begin{aligned}
& H=\sum_{k, \sigma} \varepsilon_{k} \phi_{k, \sigma}^{\dagger} \phi_{k, \sigma}+\left(\varepsilon_{d}-\mu\right) \sum_{i, \sigma} n_{i, \sigma}^{d}+U_{d} \sum_{i} n_{i, \uparrow}^{d} n_{i, \downarrow}^{d} \\
& +\frac{t_{p d}}{\sqrt{N}} \sum_{i, k, \sigma}\left(\gamma_{k} \mathrm{e}^{-\mathrm{i} k \cdot \boldsymbol{R}_{i}} \phi_{k, \sigma}^{\dagger} d_{i, \sigma}+\text { H.c. }\right)
\end{aligned}
$$

$$
\varepsilon_{k}=\varepsilon_{p}+4 t_{p p}\left|\sin \left(k_{x} / 2\right) \sin \left(k_{y} / 2\right)\right|
$$

and $\phi_{k, \sigma} \equiv \phi_{1, k ; \sigma}$, i.e., the second oxygen band, $\phi_{2, k ; \sigma}$, gets decoupled from the problem. From Eq. (11) we conclude that our problem has been reduced to the Anderson lattice model with a single conduction oxygen band. To derive the effective Hamiltonian $H_{\text {eff }}$, we apply a two-step procedure involving two approximate canonical transformations.

- The Schrieffer-Wolff (SW) [4] transformation is used to eliminate the hybridization terms.

- The usual canonical transformation [5] is used to obtain the magnetic interaction between the copper spins.

The approach we have followed up to here is similar to that considered by Ihle and Kasner [7] but they used a single fourth order canonical transformation. The effective Hamiltonian reads

$$
H_{\mathrm{eff}}=\left(\varepsilon_{d}-\mu\right) \sum_{i, \sigma} n_{i, \sigma}^{d}+\sum_{i, j} J_{\mathrm{Sx}}\left(\left|\boldsymbol{R}_{i}-\boldsymbol{R}_{j}\right|\right) \boldsymbol{S}_{i} \cdot \boldsymbol{S}_{j}
$$

where $J_{S X}\left(\left|\boldsymbol{R}_{i}-\boldsymbol{R}_{j}\right|\right)$ is the RKKY-type interaction between two spins being at copper sites $i$ and $j$, respectively. $J_{\mathrm{SX}}(R)$ is given by

$$
J_{\mathrm{SX}}(R)=\left(\frac{J}{2 N}\right)^{2} \sum_{k, k^{\prime}} n_{k} \frac{\cos \left[\left(k-k^{\prime}\right) \cdot R\right]}{\varepsilon_{k}-\varepsilon_{k^{\prime}}},
$$

where $R=\left|\boldsymbol{R}_{i}-\boldsymbol{R}_{j}\right|$. In terms of the fermion operators, the spin operators $\boldsymbol{S}_{i}$ are defined as

$$
S_{i}=\frac{1}{2} \sum_{\tau, \tau^{\prime}} d_{i, \tau}^{\dagger} \sigma_{\tau, \tau^{\prime}} d_{i, \tau^{\prime}}
$$

with $\sigma$ being a vector built up of the Pauli matrices. The constant $J=4 t_{p d}^{2} U_{d} /\left[\left(U_{d}+\right.\right.$ $\left.\left.\varepsilon_{d}-\varepsilon_{\mathrm{F}}\right)\left(\varepsilon_{d}-\varepsilon_{\mathrm{F}}\right)\right]$. Due to the presence of the occupation factor

$$
n_{k}=\left\{\begin{array}{lll}
1, & \text { if } & \varepsilon_{k} \leq \varepsilon_{\mathrm{F}}, \\
0, & \text { if } & \varepsilon_{k}>\varepsilon_{\mathrm{F}},
\end{array}\right.
$$

the expression for $J_{\mathrm{SX}}(R)$ may be further simplified yielding

$$
J_{\mathrm{SX}}(R)=\frac{J^{2}}{32 \pi^{4} t_{p p}} \int_{k_{: \varepsilon} \boldsymbol{k}^{\leq \varepsilon_{\mathrm{F}}}} \mathrm{d} k_{x} \mathrm{~d} k_{y} \int_{\mathrm{BZ}} \mathrm{d} k_{x}^{\prime} \mathrm{d} k_{y}^{\prime} \frac{\cos \left(k_{x} R\right) \cos \left(k_{x}^{\prime} R\right)}{\tilde{\varepsilon}_{k}-\tilde{\varepsilon}_{k^{\prime}}},
$$

where $\mathrm{BZ}$ denotes the integration over the Brillouin zone, $\tilde{\varepsilon}_{k}=\left|\sin \left(k_{x} / 2\right) \sin \left(k_{y} / 2\right)\right|$, and $\varepsilon_{\mathrm{F}}$ is the Fermi energy. From Eq. (17) it follows that $J_{\mathrm{SX}}(R)$ is concentration dependent. Such a dependence comes through the doping dependence of the Fermi energy $\varepsilon_{\mathbf{F}}(\delta)$. In the next section we present the numerical results obtained with the use of Eq. (17). 


\section{Approximate evaluation of the indirect magnetic interaction}

The accurate numerical calculations with the use of the full band structure will be presented elsewhere. Here we give the approximate estimates of

$J_{\mathrm{SX}}(R)$ obtained under the assumption of parabolic bands. The band energy is approximated by

$$
\sin \left(k_{x} / 2\right) \sin \left(k_{y} / 2\right) \approx \frac{1}{2 \pi} k^{2},
$$

and this gives

$$
J_{\mathrm{SX}}(R) \approx \frac{J^{2}}{16 \pi t_{p p}} \int_{0}^{k_{\mathrm{F}}} k \mathrm{~d} k \int_{k_{\mathrm{F}}}^{k_{\max }} k^{\prime} \mathrm{d} k^{\prime} \frac{J_{0}(k R) J_{0}\left(k^{\prime} R\right)}{k^{2}-k^{\prime 2}},
$$

where $k_{\max }=2 \sqrt{\pi}$ and $J_{0}$ is the Bessel function of zero order. For the numerical calculation we have chosen the values of the parameters used for the copper oxides, i.e., $t_{p d}=1.35 \mathrm{eV}, t_{p p}=0.65 \mathrm{eV}, U_{d}=8.0 \mathrm{eV}$, and $\varepsilon_{p}-\varepsilon_{d}=3.5 \mathrm{eV}[6]$. Table gives values of $J_{\mathrm{Sx}}(R)$ for $R=1$ (first neighbor), $R=\sqrt{2}$ (second neighbor), and $R=2$ (third neighbor), for three values of doping rate $\delta(0.02,0.04$, and 0.06$)$.

\section{TABLE}

Values of $J_{\mathrm{SX}}(R)$ (in $\mathrm{eV}$ ) for different doping rates $\delta$ and for $R=1, R=\sqrt{2}$, and $R=2$.

\begin{tabular}{c|c|c|c}
\hline \hline$R$ & 1 & $\sqrt{2}$ & 2 \\
\hline$\delta=0.01$ & -0.0875 & -0.0613 & -0.0427 \\
$\delta=0.02$ & -0.1344 & -0.0812 & -0.0445 \\
$\delta=0.03$ & -0.1650 & -0.0857 & -0.0340
\end{tabular}

\section{Conclusions}

We have derived the expression for the RKKY-type magnetic interaction $J_{\mathrm{SX}}(R)$, which depends on doping rate $\delta$. For the case of parabolic bands, we observe that $J_{\mathrm{SX}}(R)$ has a decaying behavior for large separation between copper ions. Our results are similar to those of Inui et al. [8] who, considering the single-band Hubbard model, obtained a doping dependent superexchange interaction by excluding double occupancies from the system.

For the high- $T_{\mathrm{c}}$ superconducting oxides, the original AF interaction (present at $\delta=0) J_{\mathrm{AF}} \approx 0.125 \mathrm{eV}$ (nonzero only for the NN copper ions). Our magnetic interaction $J_{\mathrm{SX}}(R)$ is zero for $\delta=0$ and gradually increases with increasing $\delta$ (see Table). Assuming that the original AF interaction $J_{\mathrm{AF}}$ does not change upon doping, the total magnetic interaction $J_{\mathrm{TOT}}(R)=J_{\mathrm{AF}}+J_{\mathrm{SX}}(R)$. From the approximate values given in Table, it follows that $J_{\text {TOT }}(1)$ becomes zero for a doping rate $\delta \in(0,0.04)$. It means that the long range $\mathrm{AF}$ order, present in the oxides at zero doping, should be destroyed with an increasing concentration of doped holes. The exact value of the doping $\delta_{c}$, at which the long range AF order disappears, 
is difficult to be found because there are large second- and third-neighbor interactions, $J_{\mathrm{SX}}(\sqrt{2})$ and $J_{\mathrm{SX}}(2)$, respectively. These interaction make that the oxides behave as strongly frustrated systems. With our derived expression for the magnetic interaction, we may study frustration in the way proposed by Inui et al. [8]. Finally, we would like to conclude that the presence of the $t_{p p}$ term has to be taken into account for the description of the magnetic properties of high-temperature superconductors.

\section{Acknowledgments}

JJRN and HB would like to thank CONICIT-Venezuela for financial support through the project No. F-139 and the Swiss National Foundation, project No. 4030-032788. Discussions with Dr. Terry Giamarchi, Dr. Victor Kabanov, and Prof. Andrzej M. Oleś are appreciated. In particular, Prof. Oleś carefully read the manuscript. We would like to thank Ms. María Dolores Garcia for improving the English version of the manuscript.

\section{References}

[1] P.W. Anderson, Science 234, 1196 (1987).

[2] F.C. Zhang, T.M. Rice, Phys. Rev. B 37, 3759 (1988).

[3] V.J. Emery, Phys. Rev. Lett. 58, 2794 (1987).

[4] J.R. Schrieffer, P.A. Wolf, Phys. Rev. 149, 491 (1966).

[5] B. Coqblin, J.R. Schrieffer, Phys. Rev. 185, 847 (1969).

[6] J.B. Grant, A.K. McMahan, Phys. Rev. B 46, 8440 (1992).

[7] D. Ihle, M. Kasner, Phys. Rev. B 42, 4760 (1990).

[8] M. Inui, S. Doniach, M. Gabay, Phys. Rev. B 38, 6631 (1988). 